\title{
Screening for retinopathy of prematurity in a provincial hospital in Port Elizabeth, South Africa
}

\author{
M R Jacoby, ${ }^{1}$ FCOphth; L du Toit ${ }^{2}$ FCOphth \\ ${ }^{1}$ Department of Ophthalmology, Provincial Hospital, Port Elizabeth, South Africa; Department of Ophthalmology, Faculty of Health Sciences, \\ Walter Sisulu University, Mthatha, Eastern Cape, South Africa \\ ${ }^{2}$ Department of Ophthalmology, Groote Schuur Hospital, Cape Town, South Africa
}

Corresponding author: M R Jacoby (mark.jacoby@telkomsa.net)

\begin{abstract}
Background. Retinopathy of prematurity (ROP) is an emerging public health problem in many middle-income countries where improved neonatal survival rates coupled with inadequate health resources have created a new epidemic. There are limited available data on the magnitude of the problem, and screening in South African (SA) hospitals has not been uniformly practised.

Objective. To describe the results of various interventions implemented over a 6-year period while developing a new ROP screening service in a provincial hospital in Port Elizabeth, SA.

Method. A retrospective case folder review of ROP screening at Dora Nginza Hospital, Port Elizabeth, SA, over the 6-year period 2009 2014 was conducted.

Results. A total of 919 new cases were seen. Fifteen patients received treatment for type 1 ROP (T1ROP), 223 had type 2 (T2) or earlier ROP, 1 had stage 4 ROP and 6 had stage 5 ROP. The combination of healthcare worker education, improved equipment and human resources and the introduction of dual responsibility for case referrals resulted in an increase in the number of new infants screened from 33 in year 1 to 292 in year 6. The number of infants who were screened late decreased from 33/33 (100\%) in year 1, prior to the interventions, to $23 / 292$ in the final year (7.9\%). Improved oxygen delivery and adequate oxygen saturation monitoring contributed to a decrease in the incidence of T1ROP from $1.5 \%$ to $1 \%$ over 1 year and in the incidence of T2 or earlier ROP from $30.3 \%$ to $24 \%$.

Conclusions. Better management of ROP can be achieved through adequate provision of healthcare professionals and material resources coupled with education and a well-supported referral system. A close working relationship between paediatricians and ophthalmologists results in a more efficient screening programme.
\end{abstract}

S Afr Med J 2016;106(6):598-601. DOI:10.7196/SAMJ.2016.v106i6.10663

Retinopathy of prematurity (ROP) is an important cause of childhood blindness. ${ }^{[1]}$ In the past, two epidemics occurred: the first when oxygen supplementation was used in an uncontrolled fashion in the 1950s, and the second when advances in paediatric care resulted in improved survival rates of extremely low-birth-weight babies in the 1970s. Strict oxygen administration and ROP screening with early intervention have since markedly reduced the likelihood of a poor visual outcome. ${ }^{[2,3]}$

Middle-income countries that have infant mortality rates of $\sim 50 / 100000$ population per annum are currently experiencing the so-called 'third epidemic' of ROP as a result of improved survival rates coupled with poor oxygen control and poorly established screening policies. ${ }^{[1]}$ South Africa (SA), with shortages of human resources and material resources, is at risk of developing this epidemic. ${ }^{[4]}$

ROP screening has not been the norm in many SA state hospitals, but a national ROP screening protocol was published in $2013 \cdot{ }^{[4,5]}$ ROP screening is therefore a relatively new practice in many hospitals in SA.

We describe the challenges we faced and the successes we achieved with the development of a new ROP clinic at Dora Nginza Hospital in Port Elizabeth, SA.

\section{Methods}

Clinical management from the Port Elizabeth Hospital Complex supported the study, and ethical approval was obtained through the Walter Sisulu University Research Ethics Committee (Protocol No. 043/15).

A retrospective case folder review was conducted over the 6-year period 1 January 2009 - 31 December 2014 on all infants screened for ROP. During this period, ROP screening was managed according to the following guidelines:
The criteria for ROP screening during the 6-year period were: (i) birth weight (BW) <1 $501 \mathrm{~g}$; (ii) gestational age <32 weeks; and (iii) BW $1501-2000 \mathrm{~g}$ if an unstable clinical course was reported.

The classification of disease severity was based on the revised International Classification of Retinopathy of Prematurity (ICROP) ${ }^{[6]}$ definitions. These include stage (1 - 5), zone (I - III), 'plus' disease status (absent, pre-plus or plus), and extent (clock hours). Aggressive posterior ROP (AP-ROP) is a rapidly progressive condition with prominent plus disease in a posterior location and minimal proliferative changes (Fig. 1).

The threshold for intervention was based on the Early Treatment for Retinopathy of Prematurity (ETROP) ${ }^{[2]}$ definitions. Type 1 ROP (T1ROP), defined as the presence of plus disease or stage 3 in zone I with no plus disease, was the indication for intervention. Type 2 ROP (T2ROP) was defined as zone I, stage 1 or 2 with no plus disease or zone II, stage 3 with no plus disease. These patients were observed.

Screening failures were defined as the presence of stage 4 or 5 ROP because of the poor prognosis in these cases. A successful screening programme should detect and treat patients before they develop these late stages of ROP.

The final assessment would then place the screened infant into one of three categories:

- No ROP if the retina was fully vascularised or if normal vascularisation was noted in zone III with no signs of ROP

- ROP present:

- T1 ROP

- T2 and earlier ROP

- Screening failure if stage 4 or 5 present. 
Treatment of T1ROP was laser photocoagulation of the avascular retina according to the ETROP guidelines. ${ }^{[2]}$ From 2013 onwards intravitreal bevacizumab (Avastin; Genentech) was used in cases of zone I disease according to the findings of the Bevacizumab Eliminates the Angiogenic Threat of Retinopathy of Prematurity (BEAT-ROP) study. ${ }^{[7]} \mathrm{T} 2$ and earlier cases of ROP were monitored according to the suggested schedule in the SA guidelines. ${ }^{[5]}$

\section{Data collection}

Data from patient folders were collected on:

- Number of new infants screened and number of follow-up examinations performed

- ROP screening status (recorded as either 'on time' if between 5 and 6 weeks or 'late' if after 6 weeks)

- Number of cases of ROP detected

- Clinical findings and trends. Following classification of ROP, cases were divided into: (i) T1ROP cases needing intervention; (ii) T2 and earlier ROP needing observation; and (iii) screening failures

- Management of T1ROP: indirect laser photocoagulation or intravitreal bevacizumab injections

- Additional ophthalmological problems found incidentally during ROP screening

- Complications encountered during screening

- The impact of other events and interventions related to the prevention of ROP and screening for ROP.

\section{General interventions implemented during the study period}

Screening facilities. In 2009 and 2010, the facilities for screening were inadequate. The site was separate from the neonatal unit and awareness of screening was low, as reflected by the low number of infants screened. In 2011 a new neonatal unit was built with a dedicated ROP screening room and incubator, and this helped to heighten awareness of the service.

Health professional resources and frequency of screening clinics. No ophthalmologists were based at the hospital, and the service had to be provided by the eye department at Port Elizabeth Provincial Hospital. In 2009 there were two ophthalmologists, in 2011 three ophthalmologists, and from November 2012 four ophthalmologists. The increase in the number of ophthalmologists enabled us to increase the frequency of visits from every 3 weeks in 2009 to every 2 weeks in 2011 and weekly by 2013 .

Education of staff. In 2012, ROP teaching became integrated into the paediatric academic

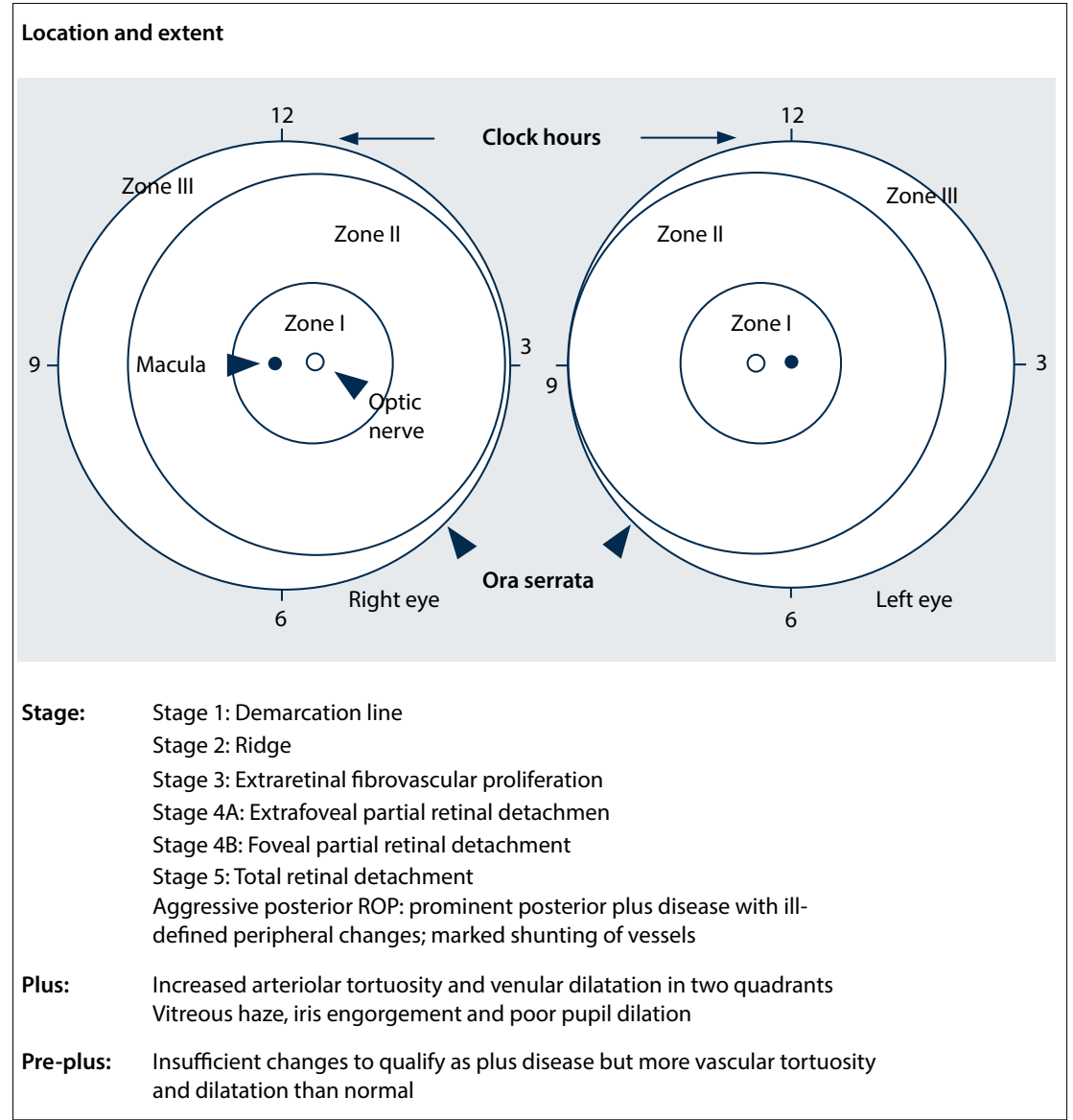

Fig. 1. The Revised International Classification of ROP (ICROP) system. ${ }^{[6]}$

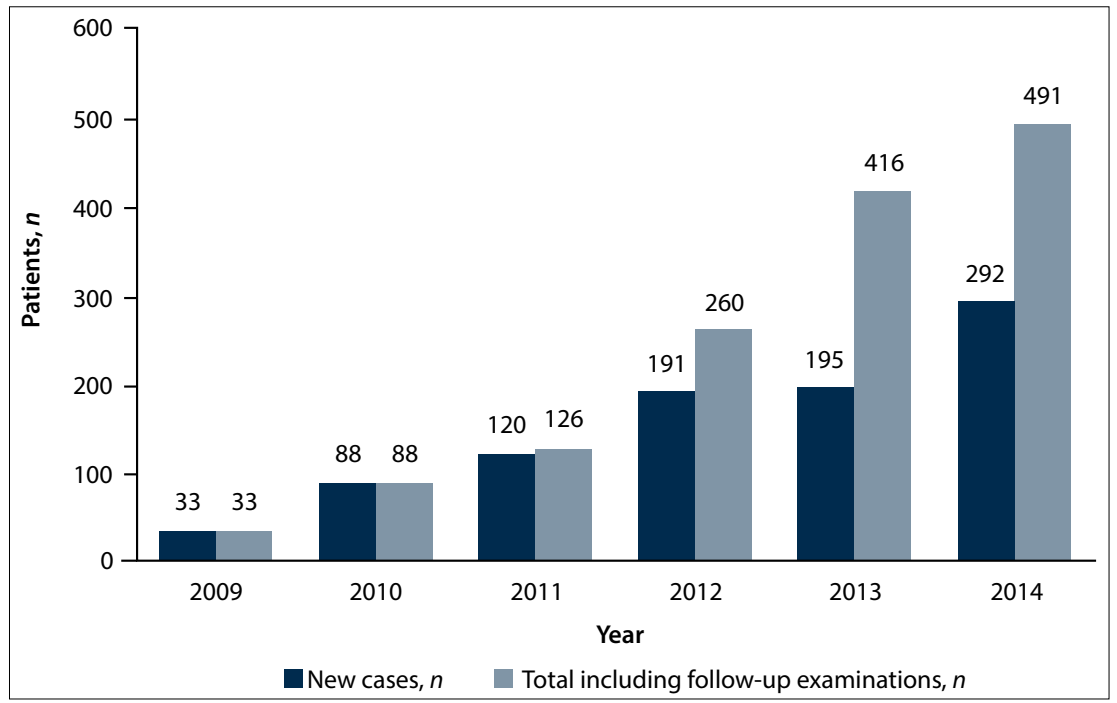

Fig. 2. Numbers of infants screened, 2009 - 2014.

programme. These sessions focused on the management of oxygen supplementation in infants and appropriate referral of infants at risk. Educational sessions for nursing staff were also implemented in 2012, and all cases of T1ROP were also discussed at the paediatric morbidity and mortality meetings from 2014 .

Responsibility for selection for screening. In 2013, we decided to augment pae- diatrician-based selection of infants with ophthalmologist selection.

Blenders and oxygen saturation monitors. There was a shortage of blenders and saturation monitors, making oxygen supplementation control impossible for most babies. In September 2013, new blenders and monitors were made available enabling better control of oxygen supplementation. 


\section{Results}

\section{Infants screened (Fig. 2)}

A total of 919 infants were screened and an additional 495 follow-up examinations were performed.

There was a progressive increase in the number of new referrals and in the total number of cases screened (new and follow-up cases combined) over the 6-year period, from 33 to 292 and from 33 to 491 , respectively.

The augmentation in 2013 of paediatrician-based selection of infants with ophthalmologist selection increased the annual number of new cases screened from 195 in 2013 to 292 in 2014

\section{Infants screened late (after 6 weeks' postnatal age)}

All infants in 2009 were referred after 6 weeks postnatal age. No data were available for 2010 - 2011. From 2012 onwards, the proportion of late referrals decreased from $51 \%$ to $33 \%$ in 2013 and to $7.9 \%$ in 2014

\section{Cases of ROP detected}

Fifteen infants had T1ROP and $223 \mathrm{~T} 2$ or earlier ROP. Of the screening failures, one infant had stage $4 \mathrm{~A}$ disease in one eye and stage 5 in the other, and 5 other infants had bilateral stage 5 ROP on presentation.

\section{Clinical findings and trends}

Table 1 sets out proportions of patients with no ROP, T1ROP and T2 and earlier ROP. The incidence of T1ROP peaked in 2012 at $2.6 \%$ and decreased to $1.0 \%$ in 2014 . T2 and earlier ROP peaked in 2013 at $30.3 \%$ and decreased to $24.0 \%$ in 2014 . This decrease followed the introduction of new oxygen blenders and saturation monitors in September 2013.

Screening failures are set out in Table 2 . All the bilaterally blind babies with stage 5 ROP presented in the first year, when all the infants were examined late. The sixth infant who developed stage 5 ROP had aggressive posterior ROP and developed stable stage $4 \mathrm{~A}$ ROP in the fellow eye after laser photocoagulation.

\section{Management and outcome of T1ROP}

Twelve infants underwent indirect laser photocoagulation and 3 had an intravitreal injection of bevacizumab. The latter treatment was reserved for zone I disease.

Two patients in the laser group developed high myopia. The infant with aggressive posterior ROP had bilateral laser photocoagulation, but developed stable stage 4A ROP in one eye and stage $5 \mathrm{ROP}$ in the other.

The infants treated with bevacizumab injections did not have any adverse events

\begin{tabular}{|c|c|c|c|}
\hline Year & $\begin{array}{l}\text { No } \\
\text { ROP }\end{array}$ & $\begin{array}{l}\text { Type } 2 \text { and } \\
\text { earlier ROP }\end{array}$ & $\begin{array}{l}\text { Type } 1 \\
\text { ROP }\end{array}$ \\
\hline 2009 & 85.8 & 0 & 0 \\
\hline 2010 & 86.0 & 13.6 & 0 \\
\hline 2011 & 74.2 & 23.3 & 2.5 \\
\hline 2012 & 68.5 & 28.3 & 2.6 \\
\hline 2013 & 68.2 & 30.3 & 1.5 \\
\hline 2014 & 75.0 & 24.0 & 1.0 \\
\hline
\end{tabular}

and did not need further injections or laser photocoagulation. One of these patients was reviewed at 17 months of age and had persistent incomplete vascularisation of zone III but no recurrence of the ROP.

\section{Additional ophthalmological problems}

Additional ophthalmological problems found incidentally during ROP screening included:

- Bilateral dense cataracts in 3 patients

- Cytomegalovirus retinitis in 1 patient

- Congenital glaucoma in 2 patients

- Presumed rubella keratitis and retinal vasculitis in 1 patient

- Transient retinal haemorrhages in 6 patients (no ROP)

\section{Complications encountered during screening}

Apnoea developed in two infants during examination. In both cases only mechanical (bag-valve-mask) ventilation was needed for less than a minute to resuscitate them, and there were no serious sequelae of these adverse events.

\section{Discussion}

The challenge of managing the increasing load of screening for ROP in middle-income countries with limited resources requires some unique solutions.

In our study, a large increase in the number of ROP examinations was achieved by: (i) having a dedicated ROP screening room and incubator; (ii) having enough ophthalmologists to ensure weekly screening; (iii) being involved in the paediatric department academic programme; and (iv) aiding the selection/referral process.

In 2014 the ratio of total to new examinations was 491:292, which represents a mean of 1.7 examinations per infant. This number could be used for planning ROP screening in other hospitals offering similar care.

In the first year of screening, five patients with untreatable bilateral stage 5 ROP were found at the first examination. Subsequent
Table 2. Screening failures (\% of new cases screened)

\begin{tabular}{lll}
\hline Year & Stage 4 & Stage 5 \\
\hline 2009 & 0 & 14.2 \\
2010 & 0 & 0 \\
2011 & 0 & 0 \\
2012 & 0.5 & 0.5 \\
2013 & 0 & 0 \\
2014 & 0 & 0
\end{tabular}

to that, there was only one child who had aggressive posterior ROP who went on to develop stage $5 \mathrm{ROP}$ in the right eye and stable stage $4 \mathrm{~A}$ ROP in the left eye despite laser treatment. This highlights the importance of timeous screening for ROP, a disease with a very narrow window period for intervention.

Two major factors played a role in reducing the incidence of T1ROP from its peak at 2.6\% to $1 \%$ by 2014 . Initially there was a shortage of oxygen blenders and oxygen saturation monitors, making it impossible to monitor every baby adequately. The acquisition of more monitors and blenders, coupled with formal education on ROP, made targeted oxygen supplementation possible. Chow et $a l .{ }^{[3]}$ showed that a very strict oxygen therapy protocol can reduce the incidence of T1ROP, and although this is only achievable in wellstaffed and well-equipped hospitals, it should be something to strive for.

Thirteen patients with other ophthalmological problems were also detected at an early stage in the screening programme. Six patients (3 with bilateral cataracts, 1 with cytomegalovirus retinitis and 2 with congenital glaucoma) needed surgical intervention or antiviral therapy.

\section{Study limitations}

A weakness of our programme and of our study is the incomplete data in the first 3 years. Accurate record keeping in the latter 3 years helped to give a better understanding of how successful our screening programme was and how the improved paediatric care had decreased the incidence of ROP.

\section{Conclusions}

If we are to halt the 'third epidemic' of ROP in SA, we need to raise awareness of the disease in hospitals that have previously not been faced with the challenge of managing babies at risk, and implement and adhere to postnatal care protocols, in particular oxygen supplementation guidelines and ophthalmic screening protocols.

In this study we demonstrated the importance of a close working relationship 
between ophthalmologists and paediatricians in setting up a new ROP programme.

\section{References}

1. Gilbert C. Retinopathy of prematurity: A global perspective of the epidemics, population of babies at risk and implications for control. Early Hum Dev 2008;84(2):77-82. DOI:10.1016/j. earlhumdev.2007.11.009

2. Good W, on behalf of the Early Treatment for Retinopathy of Prematurity Cooperative Group. Final results of the early treatment for retinopathy of prematurity (ETROP) randomized trial. Trans Am Ophthalmol Soc 2004;102:233-250.

3. Chow LC, Wright KW, Sola A. Can changes in clinical practice decrease the incidence of sever retinopathy of prematurity in very low birth weight infants? Pediatrics 2003;111(2);339-345. DOI:10.1542/peds.111.2.339
4. Varughese S, Gilbert C, Pieper C, Cook C. Retinopathy of prematurity in South Africa: An assessment of needs, resources and requirements for screening programmes Br J Ophthalmol 2008;92(7):879-882. OO:10.1136/bjo 2008.13758

5. Visser L, Singh R, Young M, Lewis H, McKerrow N (ROP Working Group, South Africa). Guideline for the prevention, screening and treatment of retinopathy of prematurity (ROP). S Afr Med J 2013;103(2):116-125. DOI:10.7196/SAMJ.630

6. Gole G, Ells A, Katz X, et al. The International Classification of Retinopathy of Prematurity revisited. Arch Ophthalmol 2005;123(7):991-999. DOI:10.1001/archopht.123.7.99

7. Mintz-Hittner H, Kennedy K, Chuang A, et al. Efficacy of intravitreal bevacizumab for stage 3+ retinopathy of prematurity. N Engl J Med 2011;364(7):603-615. DOI:10.1056/NEJMoa 1007374

Accepted 5 May 2016 